\title{
Rankings Universitarios: Calidad de las instituciones de educación superior nicaragüenses
}

University Rankings: Quality of Nicaraguan higher education institutions

\author{
Cristian Rivas-Castillo ${ }^{1}$ \\ Universidad Autónoma de Nuevo León \\ Lisbeth Velásquez Cruz ${ }^{2}$ \\ Universidad Autónoma de Nicaragua
}

\begin{abstract}
RESUMEN
El presente artículo es producto de una revisión bibliográfica, cuyo objetivo consistió en Mostrar la calidad de las universidades nicaragüenses según su posicionamiento en los principales rankings universitarios que se encargan de evaluar la calidad de las universidades, para conocer la realidad de las Instituciones de educación superior de Nicaragua. Se aplico el método de análisis, con un enfoque cualitativo, diseño no experimental, bajo un nivel documental-bibliográfico transversal. Tras la revisión documental se observó que: según las clasificaciones emitidas por el World Universities Rankings (QS), Webometrics y Scimago Institutions Rankings (SIR), no todas las instituciones de educación superior nicaragüenses son evaluadas por estos sistemas de medición, así como, que las instituciones evaluadas se posicionan en los últimos puestos. En el presente capitulo se abordará de manera general. Se concluye que existe una debilidad en la calidad de las instituciones de educación superior nicaragüenses, debido a su posicionamiento en los rankings que evalúan la calidad de estas, también, se concluye que la investigación es un factor determinante en la evaluación de la calidad del sistema educativo nicaragüense.

Palabras clave Calidad Universitaria, Docencia, Evaluación, Extensión, Instituciones de educación superior, Investigación, Rankings.
\end{abstract}

\begin{abstract}
This article is the product of a bibliographic review, and the main objective was to show the quality of Nicaraguan universities according to their positioning in the main university rankings that are responsible for evaluating the quality of the universities, in order to know about the reality of Nicaragua's higher education institutions. We used the analysis method, with a qualitative approach, non-experimental design, at cross-sectional documentarybibliographic level. After the documentary review, we noted that according to the rankings given by the World Universities Rankings (QS) Wobometrics and Scomago Institutions Rankings (SIR), not all Nicaraguan higher educations are evaluated by these measurement systems, as well as, the institutions evaluated are positioned in the latter positions. This chapter will be addressed in general way. It conclude that there is a weakness in the quality of Nicaraguan higher education institutions, due to its positioning in the rankings that evaluate the quality of these, we concluded that research is a determining factor in the evaluation of quality of Nicaraguan education system.

Key words: University Quality, Teaching, Evaluation, Extension, Higher Education Institutions, Research, Rankings
\end{abstract}

${ }^{1}$ Licenciado en Derecho por la Universidad Nacional Autónoma de Nicaragua, Maestrante en Relaciones Internacionales por la Universidad Autónoma de Nuevo León, México, Docente Investigador. Correo (Castillocris25@ yahoo.com), registro ORCID: HTTPS://ORCID.ORG/0000-0001-6827-1462

${ }^{2}$ Licenciada en Derecho por la Universidad Nacional Autónoma de Nicaragua, Magister en Derecho Procesal Civil por la Facultad de Humanidades y Ciencias Jurídicas de la Universidad Nacional Autónoma de Nicaragua, Candidata a Doctora En Métodos Alternos de Solución de Conflicto por la Universidad Autónoma de Nuevo león, México. Correo ( lilly1212@ hotmail.com), registro ORCID: HTTPS://ORCID.ORG/0000-0003-2488-2653 


\section{Introducción}

En los últimos años, el sistema de educación superior de Nicaragua ha desarrollado estrategias de evaluación y seguimiento a la calidad de sus Universidades, prestando principal énfasis a las funciones académicas y la actividad investigadora, esto con la finalidad del mejoramiento institucional, esta evaluación la cual se realiza por medio de mecanismos de medición denominados rankings de universidades. Es así, que los rankings tienen su origen en el surgimiento del Academic Ranking of World Universities (ARWU), el cual fue producido por el centro de las universidades de clase mundial (Center for World-Class Universities, CWU) de la Escuela superior de educación de la universidad Jiao Tong, de Shanghái, China en el 2003.

Es a partir de ese año que este tipo de clasificaciones se han multiplicado y sobre todo han alcanzado un impacto relevante en los foros internacionales y nacionales acerca de la evaluación de las universidades. Actualmente existen 18 rankings de evaluaciones universitarias a nivel mundial, y un número mayor de clasificaciones tanto nacionales como regionales.

En la presente Artículo se analizaron los rankings de mayor relevancia en la región latinoamericana, como lo son el World Universities Rankings (QS), Webometrics, Scimago Institutions Rankings (SIR), estos rankings utilizan indicadores y metodologías diversas, las cuales tienen niveles diferenciados de credibilidad e impacto (Ordorika, 2015).

El éxito que ha generado esta dinámica internacional no puede entenderse fuera de un contexto mundial como el actual en el cual se valora de manera preponderante las formas de clasificación y evaluación muy marcadas por la mercadotecnia, los rankings se presentan de manera más o menos explícita, como mediciones objetivas de calidad de todas las universidades del mundo.

Por tanto, en el presente trabajo se realizará una revisión documental y se abordara la importancia de la calidad en las instituciones de educación superior nicaragüense según su posicionamiento en los rankings universitarios, los cuales evalúan los indicadores de calidad de las universidades como lo son: docencia, extensión e investigación. Partiendo de esta premisa es necesario formular la siguiente interrogante: ¿cuál es la calidad de las instituciones de educación superior nicaragüenses, según su posicionamiento en los rankings QS, WEBOMETRICS Y SIR?

\section{Fundamento Teórico}

\section{Educación en Nicaragua}


De acuerdo con Tunnermann Bernheim (2008) en Nicaragua la educación se suele dividir en tres sistemas básicos: En primer lugar la Educación General básica, la cual incluye el bachillerato y la educación norma (para formar maestros de Primaria), regidos bajo la autoridad del Ministerio de Educación (MINED), el segundo sistema es la Educación profesional la cual se rige por el Tecnológico Nacional (INATEC), el tercer sistema es el de la Educación Superior, cuya instancia de coordinación es el Consejo Nacional de Universidades (CNU).

Este artículo, se centrará en las Universidades reguladas por el CNU. Este Consejo se encuentra conformado por diez Instituciones de Educación Superior (IES), siendo así que el CNU ha acreditado a 59 instituciones en todo el país (incluyendo las que conforman el Consejo). Entre las IES autorizadas se encuentran: como miembros del CNU hay 4 Instituciones Públicas, 5 Universidades privadas y un Centro de Educación Técnico Superior. Mientras que los 49 restantes son instituciones privadas acreditadas por el CNU.

\section{Calidad universitaria}

Debido al aumento de instituciones de educación superior en Nicaragua, es que se ha hecho indispensable la necesidad de evaluar la calidad de la educación universitaria, para Zegarra Rojas (2014) este concepto de evaluación, en los países latinoamericanos es relativamente nuevo y nace debido a la progresiva incorporación en políticas educacionales, para reformar la educación superior.

Por otro lado, Tunnermann (2008) indica que el concepto de calidad es como el concepto de belleza, todos sabemos o percibimos lo que es, pero encontramos difícil definirla, en este mismo sentido Bautista Arríen (1995) señala que la calidad de la educación superior es una especie de utopía, estas aproximaciones conceptuales de la calidad revelan la dificultad de poder tener un solo concepto o una sola visión de este tema.

Esta diversidad de conceptos también se encuentra basados en distintos aspectos, siendo estos el de calidad como excelencia, en cual se busca que las universidades posean estudiantes sobresalientes, académicos destacados y aseguramiento de primer nivel, sin embargo, esta conceptualización de la calidad está diseñada para ser aplicada en una educación de elite, algo que está muy alejada de la realidad de los países latinoamericanos (Águila Cabrera, 2005).

En otro sentido, señala Águila Cabrera (2005) es la calidad como respuesta a los requerimientos del medio, la cual se basa en una definición de prima pertinencia, pero trae el peligro de que la calidad se tome solamente sobre la base de lo que se requiere, haciendo que las instituciones abandonen su misión universitaria. 
También, la Organización de las Naciones Unidas Para la Educación, la Ciencia y la Cultura (UNESCO) señala que la calidad es un concepto multidimensional, que abarca todas las funciones y actividades principales de las universidades; calidad de enseñanza, de formación e investigación, lo cual significa, la calidad de su personal docente, administrativo, estudiantil y de infraestructura.

Por lo tanto, los sistemas universitarios están conformados por tres procesos básicos: docencia, investigación y extensión, la evaluación de la calidad de estos sistemas es mucho más compleja que la de otros niveles del sistema educativo, debido a que la educación en las universidades es multifuncional.

En este sentido, la medición de la calidad conlleva a que las universidades se sometan a un proceso, donde se evalué la calidad de su docencia, investigación, servicios, internacionalización y organización, esto con el objetivo de ser reconocidas tanto a niveles internos como externos.

Al mismo tiempo, Reyes (2016) expresa que la noción de calidad universitaria se ha instalado en el mundo por medio de diferentes sistemas de evaluación y medición que la operacionalizan a través de criterios e indicadores respecto de ciertos aspectos de la universidad, dentro de estos sistemas de evaluación se encuentran los rankings universitarios, los cuales son desarrollados por entidades no estatales

\section{Rankings universitarios}

Los rankings universitarios tienen como objetivo establecer un orden jerárquico de las instituciones de educación superior, basados en parámetros e indicadores que midan la calidad de la educación universitaria (Albornoz \& Osorio, 2017) en este sentido PerezEsparrells \& Gómez-Sancho (2010) establece que los rankings tratan de establecer comparaciones entre universidades según criterios de calidad.

Estos mecanismos de medición surgen precisamente por el interés de evaluar la calidad de la formación y la investigación que se desarrollan en las universidades (Tomas Folch, Mónica Feixas, Bernabéu-Tamayo, José Ruiz, 2015), además, sirven como instrumentos para los Estados de analizar sus políticas educativas.

El éxito de estos mecanismos de medición se debe a que la sociedad globalizada del conocimiento, demanda mayor calidad por parte de las instituciones de educación superior, es por esta razón que los rankings se basan en indicadores de opinión e indicadores bibliométricos/ciber métricos (Kruger y Molas, 2010).

Los rankings universitarios tradicionalmente se ordenan por países, regiones, globales y 
especializados (Barsky, 2014), las clasificaciones por países son aquellas que se centran en el desempeño de las universidades en su contexto nacional, los rankings globales, que suelen conocerse como mundiales o internacionales, realizan una cobertura más amplia debido a que estudian comparativamente a universidades de todo el mundo (Aguillo, 2012).

\section{Principales rankings de universidades}

A continuación, se expondrán las principales características de los rankings universitarios internacionales donde se evalúan a las universidades nicaragüenses, se analizarán los criterios de medición y el posicionamiento de las instituciones.

\section{WORLD UNIVERSITY RANKINGS (QS)}

El QS es un ranking realizado por la firma Quacquarelli Symonds publicado anualmente por la revista Times Higher Education magazín (THE), considerado uno de los más prestigiosos y reconocidos a nivel mundial, el cual tiene como objetivo principal el proveer a la comunidad universitaria un mecanismo de evaluación comparativa de sistemas de educación superior. En la tabla 1, se muestran los criterios que utiliza el ranking QS para emitir sus calificaciones.

Tabla 1: Criterios de evaluación QS

\section{Criterios}

Reputación Académica

Reputación del Empleador

Proporción de profesores por alumno

Personal con doctorado

\section{Conceptos}

Evalúa las percepciones de académicos de todo el mundo con respecto a la calidad de la enseñanza y la investigación en las mejores universidades.

Pide a los empresarios que identifiquen las instituciones de las cuales obtienen los graduados más competentes, innovadores y eficaces.

Un mayor número de docentes por alumnos es un indicador indirecto del compromiso de las instituciones con la enseñanza de alta calidad.

Evalúa la calidad de la capacitación del personal académico, detectando la proporción de ellos que han alcanzado el nivel más alto de educación en su área de especialización. 
Red de investigación internacional

Citas por papel

Trabajos por facultad

Impacto web
Utiliza los datos proporcionados por Scopus, evalúa el grado de apertura internacional en términos de colaboración de investigación para cada institución evaluada.

Mide el número promedio de citas obtenidas por publicación y es una estimación del impacto y la calidad del trabajo científico realizado por las universidades, solo se evalúan las instituciones que producen más de 150 artículos en los últimos cinco años.

Determina el numero promedio de publicaciones científicas (artículos) producidas por facultad y evalúa la productividad de la investigación de las instituciones, se utilizan documentos indexados de cinco años completos.

Evalúa la efectividad con que las instituciones están haciendo uso de las nuevas tecnologías.

Fuente: elaboración propia (2019).

La reputación académica es la ponderación más alta que tiene este ranking, a la cual se le asigna un valor equivalente al $40 \%$ del total, este indicador recopila las opiniones de los expertos de más de 80,000 personas en el espacio de educación superior sobre la calidad de la enseñanza y la investigación en las universidades del mundo. La reputación del empleador representa el $10 \%$ de la puntación total, este se basa en una encuesta que se realiza a empleadores a nivel mundial, el cual tiene como propósito identificar a las universidades que producen los mejores graduados para el mercado laboral.

También, se encuentra el indicador proporción Facultad/ Estudiante, tiene como objetivo medir la estructura organizacional de la institución, esta evaluación se realiza utilizando un radio en el cual se tiene en cuenta el número de profesores que hay por cada estudiante que se encuentra matriculado en la institución, este apartado tiene un valor del $20 \%$ y demuestra cual institución está mejor equipada para proveer una mejor supervisión y asesoría a sus pupilos.

Por otro lado, se evalúa la investigación siendo que esta es una de la misión más importante que tienen las universidades, es por tal razón que el ranking mide la calidad de la 
investigación institucional utilizando su métrica de citas por facultad, las cuales se calculan tomando en cuenta el número de citas recibida por todos los documentos producidos por una institución en un periodo de cinco años por el número de miembros de la facultad de esa institución, los documentos se extraen de la base de datos Scopus, es decir, los documentos estudiados solo podrán ser aquellos que las universidades o sus investigadores publiquen en esta prestigiosa plataforma, este indicador tiene una ponderación del $20 \%$.

La proporción de docentes internacionales y proporción de estudiantes internacionales tienen en valor cada una del 5\%, este indicador mide directamente la función de internacionalización de las universidades, se toman en cuenta estudiantes que estén matriculados en la institución, así como aquellos que se encuentran realizando intercambio académico o bien estancias de investigación.

Para este estudio se analizó el ranking correspondiente a la región latinoamericana del año 2019, donde se evaluaron un total de 391 instituciones de educación superior, ocupando el primer lugar de la región la Pontificia Universidad Católica de Chile (UC), en el caso de Nicaragua, solo fueron evaluadas tres universidades siendo estas, la Universidad Centroamericana (UCA), Universidad Nacional Autónoma de Nicaragua (UNAN-Managua) y la Universidad Nacional de Ingeniera (UNI).

En este sentido, se observó que en los últimos 3 años solo estas instituciones nicaragüenses antes mencionadas han sido tomadas en cuenta para ser evaluadas, siendo que son las únicas que cumplen con los indicadores que establece el QS para poder ser clasificadas, aunque sus puntuaciones las sitúan en los últimos puestos del ranking correspondiente a la región latinoamericana.

Es así, como la universidad centroamericana en la clasificación correspondiente al año 2019 experimento un descenso en relación con los puestos que ocupo en el año 2018 y 2017, en el último ranking emitió se sitúa en la posición 301, y en las dos clasificaciones anteriores se mantuvo en el puesto 251, siendo así la segunda mejor institución del país.

En este mismo sentido, la universidad Nacional Autónoma de Nicaragua, UNANManagua, es la que mejor se ha ubicado en las últimas clasificaciones emitidas, en las ediciones correspondientes a los años 2017 y 2018 se situó en el puesto 201, sin embargo, en el informe del año 2019 aparece posicionada en el lugar 251.

Por último, la universidad Nacional de Ingeniería, es la tercer Institución Nicaragüense que aparece en la clasificación emitida por QS, ocupando los últimos puestos de la evaluación en el ranking correspondiente al año 2019 se posiciona en el sitio 351 de 391 universidades evaluadas. 


\section{Webometrics}

El Ranking Web de Centros de Investigación del Mundo, es elaborado por el Laboratorio de Cibermetria perteneciente al Instituto de Bienes y Políticas Públicas (IPP), el cual realiza un análisis cuantitativo de internet y los contenidos de la red de las instituciones académicas que se encuentran relacionadas con el proceso de generación y comunicación académica del conocimiento científico. En la tabla 2, se muestran los criterios establecidos por el Webometrics:

Tabla 2: Webometrics

\begin{tabular}{lc}
\hline INDICADORES & CONCEPTO \\
Tamaño (S) & Número de páginas obtenidas a partir \\
& de 4 motores de búsqueda: Google, \\
& Yahoo!. Live search y exalead. \\
Visibilidad (v) & Número total de enlaces externos \\
& recibidos. \\
Scholar (Sc) & Google académico provee el número \\
& de artículos y citaciones de cada dominio \\
& académico. \\
\hline
\end{tabular}

Fuente: elaboración propia (2019).

El objetivo principal de esta institución es el de convencer a todos los miembros de las comunidades académicas y políticas de la importancia de la publicación web, esto como una forma de que las instituciones midan su actividad científica, para la elaboración del ranking se utilizan indicadores bibliométricos y ciencimetricos.

Estos indicadores permiten medir la actividad y visibilidad de las universidades, esta evaluación se realiza por medio del análisis del volumen de la información que las instituciones publican y el número de enlaces que otras webs tienen hacia las que son analizadas.

Es decir, el ranking WEBOMETRICS permite conocer la calidad en docencia que poseen las universidades, así como, el que hacer de las mismas en relación a la investigación, siendo esta uno de los indicadores claves en la realización de la clasificación, ya que se analizan la cantidad de artículos que las universidades están publicando.

En su última actualización, la plataforma analizo un total de 3695 universidades latinoamericanas, siendo la Universidad de Sao Paulo USP la mejor evaluada a nivel regional, por Nicaragua 42 universidades fueron estudiadas, sin embargo, en el presente artículo se mencionarán las tres mejores universidades del país según el ranking WEBOMETRICS. 
En este sentido, la Universidad Nacional Autónoma de Nicaragua, UNAN-Managua, es la institución número 1 a nivel del país y el lugar 400 en el continente, seguida de la Universidad Nacional Autónoma de Nicaragua, UNAN-León y la Universidad Centroamericana (UCA).

\section{Scimago Institutions Rankings (SIR)}

El Scimago Institutions Rankings es una clasificación de las instituciones de educación superior que se basa principalmente en la producción científica de los últimos cinco años (SCIMAGO, 2017), es uno de los instrumentos más importantes en Latinoamérica debido a que ha servido de instrumento para facilitar el análisis en la toma de decisiones para el fortalecimiento y desarrollo de la investigación en las Universidades de la región.

Para la evaluación de las universidades, el SIR cuenta con múltiples indicadores bibliométrica que han sido discutidos por la comunidad científica, lo cual permite determinar el desempeño, calidad y pertinencia de la producción investigativa, los criterios de evaluación del SIR se detallan en la Tabla 3.

Tabla 3: Criterios SIR

\begin{tabular}{|c|c|c|}
\hline \multirow{12}{*}{$\begin{array}{c}\text { FACTOR } \\
\text { Investigación }\end{array}$} & INDICADOR & CONCEPTO \\
\hline & Excelencia con liderazgo (EwL) & Indica la cantidad \\
\hline & & documentos en excelencia en los \\
\hline & & que las instituciones es el \\
\hline & & principal contribuyente. \\
\hline & Impacto normalizado (NI) & Se calcula sobre la producción \\
\hline & & $\begin{array}{l}\text { de liderazgo de la institución, } \\
\text { muestra la relación entre el } \\
\text { impacto científico promedio de } \\
\text { una institución y el promedio } \\
\text { mundial establecido. }\end{array}$ \\
\hline & Salida $(0)$ & documentos \\
\hline & & en revistas \\
\hline & & académicas indexadas en Scopus. \\
\hline & Grupo de talento científico (STP) & $\begin{array}{l}\text { Total, de autores diferentes de } \\
\text { una institución en el resultado }\end{array}$ \\
\hline & & $\begin{array}{l}\text { total de publicación de esa } \\
\text { institución durante un periodo de } \\
\text { tiempo determinado. }\end{array}$ \\
\hline
\end{tabular}


Liderazgo científico (L)

Colaboración Internacional (IC)

Excelencia (Exc)

Innovación

Impacto Social

Patentes (PT)
Indica la cantidad de producción de una institución como contribuyente principal, es decir, la cantidad de documentos en los que el autor correspondiente pertenece a la institución.

Producción de la institución producida en colaboración con instituciones extranjeras.

Publicaciones de alta calidad (Q1) Cantidad de publicaciones que una institución pública en las revistas académicas más influyentes del mundo.

Indica la cantidad de producción científica de una institución que se incluye en el $10 \%$ superior de los trabajos más citados en sus respectivos campos.

Conocimiento Innovador (IK) Resultado de una publicación científica de una institución citada en las patentes.

Porcentaje de la producción de publicaciones científicas citada en las patentes.

Número de solicitudes de patentes.

Número de páginas asociadas a la URL de la institución según Google.

Numero de redes de retroceso Numero de redes (subredes) $(\mathrm{BN})$ de las que provienen los enlaces entrantes al sitio web de la institución.

Fuente: elaboración propia (2019).

En la clasificación correspondiente al año 2019 esta plataforma analizo un total de cuatrocientas instituciones de Latinoamérica, lo cual significó un aumento de cuarenta y cinco universidades en relación al año 2018, donde se evaluaron trescientos cincuenta y cinco 
centros de investigación, siendo la Universidad de Sao Paulo (Brasil) el centro mejor evaluado en las dos últimas ediciones.

Se realizó un análisis de las clasificaciones emitidas desde el año 2009 hasta la actualidad, con la finalidad de conocer la realidad de las instituciones de educación superior de Nicaragua, con lo cual se logró apreciar la ausencia de estas instituciones en el SIR, esto debido a que este instrumento de medición de la calidad universitaria en su aspecto de la investigación toma como parámetro las publicaciones que las Instituciones de educación superior realizan en la plataforma Scopus en un periodo de cinco años.

Scopus es una base de datos bibliográfica, en la cual se colocan documentos de las áreas de ciencia, tecnología, medicina, ciencias sociales, artes y humanidades, es decir, es la plataforma de mayor prestigio y calidad dentro de la comunidad científica, para conocer la actividad de investigación de las universidades nicaragüenses se hizo una búsqueda exhaustiva de los documentos publicados por estos centros de enseñanza, en lo cual se puede constatar la poca actividad en investigación de las instituciones de educación superior del país centroamericano.

Esto no significa que en Nicaragua no se realiza investigación de calidad, sino, que esta actividad está siendo realizada por Universidades y centros de investigación extranjeros, como se puede observar en la imagen 1:

Imagen 1: Publicaciones Scopus realizadas en Nicaragua.

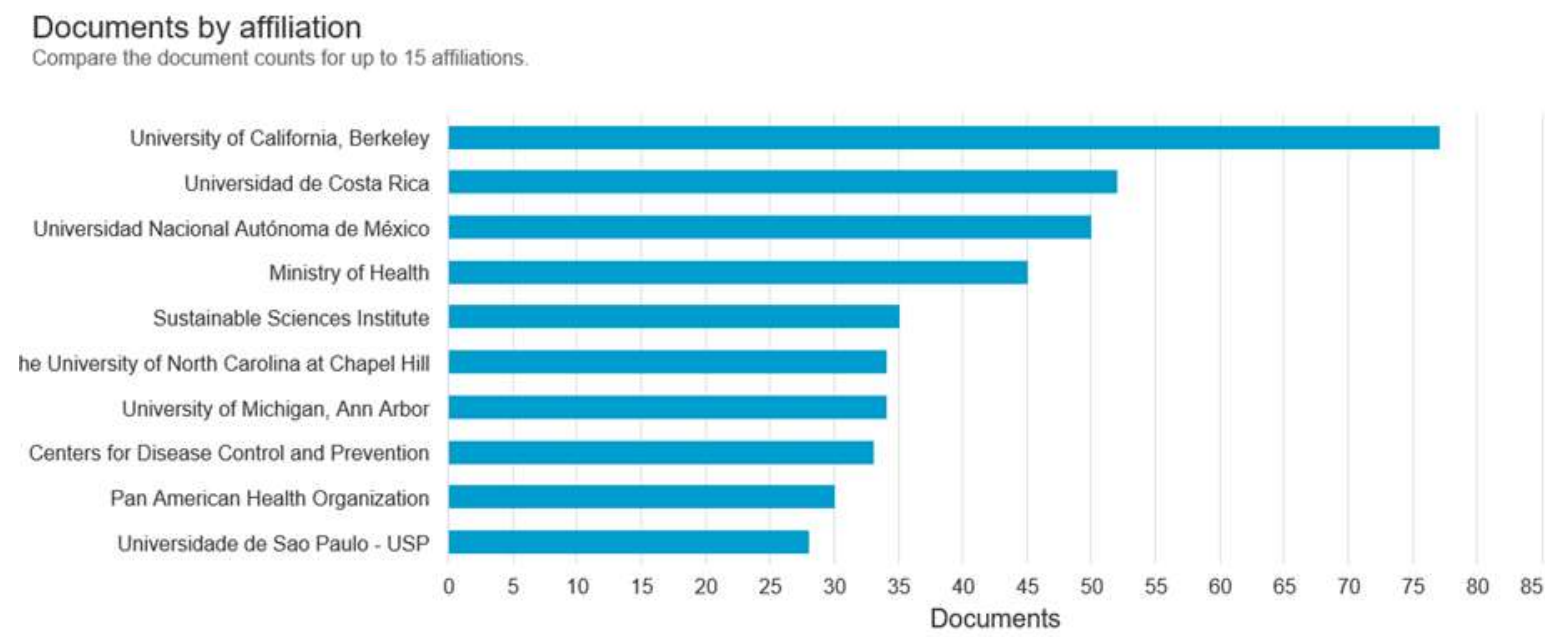

Fuente: Scopus (2019).

En este sentido, se buscaron documentos almacenados en la base de datos Scopus por filiación institucional, es decir, documentos publicados por docentes o investigadores de las instituciones de educación superior nicaragüenses, en total se encontraron 15 instituciones que realizan investigaciones relacionadas con Nicaragua o investigación en el país, estas 
instituciones son de procedencia extranjera, también, se observó la ausencia de universidades nicaragüenses dentro de la plataforma.

Siendo así, la universidad de california, Berkeley la universidad que más aporta documentos en temas relacionados con Nicaragua o investigaciones realizadas dentro del territorio nicaragüenses con un total de setenta y siete documentos en un periodo de cinco años, seguido de la Universidad de Costa Rica, la cual suma en el mismo periodo de tiempo cincuenta y dos documentos, y la Universidad Nacional Autónoma de México realizo cincuenta investigaciones que fueron publicados en la plataforma Scopus.

\section{3.- MÉTODO}

\section{Diseño}

El enfoque investigativo de la presente investigación es cualitativo, de acuerdo con Hernández, Batista y Fernández (2014) "Utiliza la recolección y análisis de los datos para afinar las preguntas de investigación o revelar nuevas interrogantes en el proceso de interpretación" (p.7).

Se utilizó la técnica de análisis de contenido, siendo esta "una técnica de investigación para la descripción objetiva, sistemática y cuantitativa del contenido manifiesta de la comunicación” Berelson citado en (Rodríguez-Burgos \& Rivas Castillo, 2018).

Alcanzando un diseño no experimental "Que se realiza sin la manipulación deliberada de variables y en los que sólo se observan los fenómenos en su ambiente natural para después analizarlos" (Hernández, Batista y Fernández, 2014, p. 149).

El alcance establecido es el exploratorio "emplean cuando el objetivo consiste en examinar un tema poco estudiado o novedoso" (Hernández, Batista y Fernández, 2014, p. 91).

\section{Instrumentos}

Para la construcción del marco teórico-conceptual de la temática "Rankings universitarios: Calidad y posicionamiento de las instituciones de educación superior nicaragüenses", se consultaron un total de diecinueve referencias bibliográficas utilizándose como instrumento las ideas, argumentos y proyectos que fueron interpretados desde una perspectiva analítica y crítica.

\section{Procedimiento}

Con relación a la comprensión del problema de la investigación se recopilan fuentes secundarias de documentos académicos. En el marco de referencia se definen los conceptos básicos relativos a la calidad universitaria y rankings de universidades. Una vez recopilada y analizada la información se construye el documento objeto de este trabajo. Por último, se 
realizan las recomendaciones y conclusiones conforme a los objetivos trazados (Bascón et al, 2016, p. 39).

\section{Conclusiones}

La calidad universitaria, se debe de analizar desde una perspectiva holística tomando en cuenta los tres sistemas fundamentales que toda institución de educación superior debe de poseer, esto sistemas son la docencia, investigación y extensión, la ausencia de uno de estos tres aspectos presupone, la falta de calidad de las universidades.

Para conocer el grado de calidad que posee cada institución de educación superior, se ha recurrido en los últimos años a los rankings universitarios, los cuales se encargan de evaluar el desempeño de las IES y emitir clasificaciones que le permiten a la sociedad, a los Estados y a las propias Universidades conocer la realidad del desempeño de sus funciones académicas, estos rankings dan oportunidad a proponer mejoras a los sistemas de educación superior de los países.

En este artículo se analizaron tres rankings universitarios, cada uno evalúa un sistema específico de la calidad universitaria, el QS mide la función docencia, Webometrics la función extensión y el SIR la función investigación. En este sentido, en el Rankings QS, se encontraron tres universidades de Nicaragua las cuales se sitúan en las últimas posiciones de las instituciones de educación superior de Latinoamérica que fueron evaluadas, en el Rankings Webometrics se posicionan un total de cuarenta y dos universidades nicaragüense teniendo una puntación baja respecto a sus homologas de la región, y en el Rankings SIR, el cual evalúa la función investigación que realizan las universidades, se pudo observar la ausencia de los centros de enseñanza de Nicaragua.

Por último, la ausencia en el principal ranking a nivel latinoamericano que evalúa la función de investigación significa una debilidad en la calidad universitaria nicaragüense, debido a que no se está realizando producción científica, esto no quiere decir que existe una falta de investigación, sino, que estas investigaciones realizadas no están culminando el proceso de divulgación, lo cual sería la publicación de sus trabajos en las principales bases datos como Scopus. 


\section{Referencias}

AGUILLO, I. (2012): "Rankings de Universidades: El Ranking Web", Higher Learning Research Communication. Vol., 2, 3-22, Recuperado a partir de: www.hlrcjournal.com/index.php/HLRC/article/download/56/64.

Águila Cabrera, V. (2005). "El concepto calidad en la educación universitaria: clave para el logro de la competitividad institucional". Revista Iberoamericana De Educación, 36(12), Recuperado a partir de https://rieoei.org/RIE/article/view/2886.

Albornoz, M. Osorio, L. (2017) "Uso Público de la Información: el caso de los rankings de universidades". Revista Iberoamericana de ciencia, tecnología y sociedad. (Centro Redes), Vol. 12, 11-49.

Bautista Arríen, J. (1995). "Reflexiones Sobre la Educación". Instituto Nicaragüense de Investigación y Educación Popular (INIEP) y UNESCO, Managua. p. 5.

BARSKY, O. (2014): "La evaluación de la calidad académica en debate: los rankings internacionales de las universidades y el rol de las revistas científicas", Buenos Aires, Ed. Teseo, Universidad Abierta Interamericana.

Krüger, K. \& Molas, A. (2010). "Rankings mundiales de universidades: objetivos y calidad". Ar@cne. Revista Electrónica de Recursos en Internet sobre Geografía y Ciencias Sociales. Recuperado de: http://www.ub.es/geocrit/aracne/aracne-129.htm.

Hernández, R., Fernández, C., y Baptista, P. (2014). Metodología de la investigación. México: Mc Graw Hill.

Lechuga, J. (2018). Necesidades insatisfechas de una comunidad aledaña a una terminal portuaria de barranquilla. Revista Pensamiento y Gestión, 45 (2) 181-210.

Ordorika, I. (2015). Rankings universitarios. Revista De La Educación Superior, 44(173), 7 9. Recuperado a partir de http://resu.anuies.mx/ojs/index.php/resu/article/view/171

Pérez-Esparrells, C. \& Gómez Sancho, J. (2010). "Los rankings internacionales de las instituciones de educación superior y las clasificaciones universitarias en España: visión panorámica y prospectiva de futuro", Biblioteca Universidad Autónoma de Madrid, paginas 1-54.

Quacquarelli, S. (QS). (2018) obtenido de: https://www.topuniversities.com/universityrankings/latin-american-university-rankings/2019.

Ranking Web of Universities (WEBOMETRICS) http://www.webometrics.info/en.

Reyes, C. (2016). "Medición de la Calidad Universitaria en Chile: La Influencia de la Rankings", Calidad en la Educación, Consejo Superior de Educación Marchant Pereira, No. 44, 158-196.

Rodríguez-Burgos, Karla, Rivas-Castillo, Cristian. (2018). Propuesta de instrumento para evaluar la producción científica de las universidades en Centroamérica. Revista Legem Vol.4 Num.2. pp.1-20.

Scimago Institutions Rankings (SIR) www.scimagoir.com/rankings.php. 
Tomas Folch, M. Feixas, M. Bernabéu-Tamayo, M. Ruiz, J. (2014). "La literatura científica sobre rankings universitarios: una revisión sistemática" Revista de docencia Universitaria (REDU), Vol., 13(3), 33-54.

Tunnermann Bernheim, C. "Calidad, Evaluación Institucional, Acreditación y Sistemas Nacionales de Acreditación. Instituto Latinoamericano de Educación para el Desarrollo.

Tunnermann Bernheeim, C. (2008) "La educación superior en Nicaragua" Revista de Avaliacao de Educacao Superior, Universidad Estadual de Campinas, Vol.13, 337367.

Zegarra Rojas, O. (2014). "Calidad, Evaluación y Acreditación Universitaria". Acta Herediana. Universidad Peruana Cayetano Heredia, Vol. 54, 45-53. 